\title{
Low acceptability of medical male circumcision as an HIV/AIDS prevention intervention within a South African community that practises traditional circumcision
}

\author{
Daniella Mark, Keren Middelkoop, Samantha Black, Surita Roux, Llewellyn Fleurs, Robin Wood, Linda-Gail Bekker
}

Background. Traditional circumcision is practised among some indigenous tribes in South Africa (SA) such as the Xhosa. Recent experimental evidence has demonstrated the benefits of male circumcision for the prevention of HIV infection in heterosexual men. The acceptability of circumcision as a biomedical intervention mirroring an ingrained cultural practice, as well as the age and extent of the procedure, are poorly understood.

Methods. Men aged 15 - 42 years were recruited in a peri-urban settlement near Cape Town. Participants completed an intervieweradministered questionnaire assessing self-reported circumcision status, context and reasons for previous or planned circumcision, and willingness to undergo medical circumcision for themselves or their sons. Results were confirmed by clinical examination. The most recent HIV test result was compared with circumcision status.

Results. Of the 199 men enrolled, 148 (74\%) reported being traditionally circumcised; of the 51 not circumcised, 50 were planning the traditional procedure. Among men self-reporting circumcision,
40 (27\%) had some or all of the foreskin remaining. The median age at traditional circumcision was 21 years (interquartile range $19-22$ years). While knowledge of the preventive benefit of circumcision was reported by 128 men (66\%), most were unwilling to undergo medical circumcision or allow their sons to do so, because of religion/culture, notions of manhood, and social disapproval.

Conclusion. Almost all men in this study had undergone or were planning to undergo traditional circumcision and were largely opposed to the medically performed procedure. In the majority, traditional circumcision had occurred after the mean age of sexual debut and almost a quarter were found to have only partial foreskin removal. To ensure optimal HIV prevention benefits, strategies to facilitate complete foreskin removal prior to sexual debut within traditional circumcision practices require further attention.

S Afr Med J 2012;102(6):571-573.
In 2009 there were 5.7 million people with HIV in South Africa (SA), accounting for $18 \%$ of all infections internationally. ${ }^{1}$ Although prevalence rates are higher in women, 1 in $4(25.8 \%)$ men aged 30 - 34 years is infected. ${ }^{2}$ Despite significant steps towards universal access to antiretroviral therapy, the ever-increasing number of HIV-infected South Africans makes uptake and improvement of prevention interventions urgent.

Male circumcision is an effective prevention tool for reducing female-to-male HIV transmission. Randomised controlled trials conducted in SA, Kenya and Uganda have reported point efficacies of $60 \%, 53 \%$ and $51 \%$, respectively. ${ }^{3-5}$ Modelled data from 2006 have shown the potential population impact of circumcision rollout, ${ }^{6}$ projecting the prevention of approximately 2 million new HIV infections and 300000 AIDS deaths in sub-Saharan Africa in the next 10 years. Additional health benefits associated with the procedure include a lower risk of other sexually transmitted infections (STI) such as chancroid and syphilis. ${ }^{7}$

For medical circumcision (MC) to be effective as an HIV prevention measure, it will need to be acceptable and accessible to young

Desmond Tutu HIV Centre, Institute of Infectious Disease and Molecular Medicine and Department of Medicine, University of Cape Town

Daniella Mark, BSocSc, MA, $\mathrm{PhD}$

Keren Middelkoop, $\mathrm{MB} \mathrm{ChB}, \mathrm{PhD}$

Samantha Black, BAppSc (Pthy), MPH

Surita Roux, MB ChB, MPH

Llewellyn Fleurs, $\mathrm{MB} \mathrm{ChB}$

Robin Wood, BM BCh, DTM\&H, MMed, FCP (SA)

Linda-Gail Bekker, MB ChB, DCH, DTM \& H, FCP (SA), PhD men in SA and should take place before sexual debut. A national survey found that the majority of circumcised men aged 15 years or older had been circumcised traditionally $(57 \%) .{ }^{8}$ Within the Xhosa tribe, who comprise $23 \%$ of black South African men, ${ }^{9}$ traditional circumcision (TC) is considered a 'rite of passage to manhood. ${ }^{10}$ The age and extent of foreskin removal in TC, and the acceptability of replacing the procedure with $\mathrm{MC}$, is poorly understood.

We investigated a cohort of mainly Xhosa men to determine the proportion who had undergone TC, the extent of foreskin removal, and attitudes towards MC as an HIV prevention tool, for themselves and their sons.

\section{Methods}

The study was conducted at an HIV prevention trial site in a periurban settlement close to Cape Town, SA, which has an estimated population of $350000,{ }^{11}$ an HIV prevalence of $23 \%{ }^{12}$ and a $99 \%$ black African and 95\% Xhosa-speaking demographic. ${ }^{11}$

Volunteers were eligible for the study if they were male, aged 15 - 42 years, and living in the settlement at the time of the study. Participants were recruited from an observational cohort and the voluntary counselling and testing (VCT) service and were sequentially enrolled. Volunteers were offered regular HIV and STI screening as well as risk reduction counselling. All study participants gave written informed consent and the study was approved by the University of Cape Town's Research Ethics Committee.

Demographic and screening data were kept in a central database. Participation required a single study visit, wherein volunteers were screened for eligibility and enrolled. Participants completed an interviewer-administered questionnaire assessing self-reported circumcision status, context of and reasons for previous or planned circumcision, and willingness to undergo $\mathrm{MC}$ and/or willingness to permit a son's MC. Recognising that TC comprises several cultural components, an alternate term, 'removal of foreskin', was 
used in addition to $\mathrm{MC}$, implying only the surgical procedure. The questionnaire was administered in isiXhosa by a trained interviewer.

Participants underwent a visual genital examination by a medical officer, after which the medical officer completed a circumcision assessment form to classify participants as uncircumcised (no evidence of surgery), partially circumcised ( $>40 \%$ but $<100 \%$ foreskin remaining), partially circumcised ( $>0 \%$ but $<40 \%$ foreskin remaining) or completely circumcised (no foreskin remaining). Participants' most recent HIV test result was drawn from their last HIV test from the observational cohort or their VCT result, with $53 \%$ of participants recruited on the same day as their HIV test (median 135 days, interquartile range (IQR) 0 - 777 days between test and recruitment).

Data were analysed using STATA 10.0 software. Bivariate analyses employed chi-square and Wilcoxon rank sum tests, as appropriate. Logistic regression models were developed to examine factors associated with self-reported and medically assessed circumcision status. Kappa statistics were calculated to determine the degree of agreement between self-reported and medically assessed circumcision status. The 95\% confidence intervals (CI) were based on the Poisson distribution and all statistical tests were two-sided at $\alpha=0.05$.

\section{Results}

One hundred and ninety-nine participants were enrolled between March 2009 and February 2010. The baseline characteristics of the cohort are represented in Table 1 . The median age of participants was 28 years (IQR 22 - 35 years). One hundred and six (53\%) identified themselves as Christian, $53(27 \%)$ as belonging to a traditional African religion, and the remaining $40(20 \%)$ as not belonging to a religious group. One hundred and fifty-one (76\%) were born in Cape Town, 44 (22\%) in the Eastern Cape, 3 elsewhere in SA, and 1 in Lesotho. All were black South Africans and spoke isiXhosa.

Among those who reported having been circumcised and those planning to be circumcised, there was no statistically significant difference in willingness to undergo $\mathrm{MC}$ or surgical removal of their foreskins ( $p=0.99$ and $p=0.23$ respectively). Therefore, in the remainder of this paper, surgical procedure and medical circumcision are termed the same - medical circumcision (MC).

Of the total, 148 participants (74\%) self-reported having been circumcised; of the remaining 51 participants, 50 were planning to be circumcised in the future. The single participant not planning circumcision was a 34-year-old, who reported not belonging to a religious group. One participant who reported having been circumcised subsequently

Table 1. Baseline characteristics of participants $(N=199)$

\begin{tabular}{ll}
\hline & $\boldsymbol{n}(\%)$ \\
\hline Birthplace & $151(76)$ \\
Cape Town & $44(22)$ \\
Eastern Cape & $4(2)$ \\
Other & \\
Religion & $53(27)$ \\
Traditional African & $106(53)$ \\
Christian & - \\
Muslim & $40(20)$ \\
None & - \\
Other & \\
Circumcision status & $148(74)$ \\
Circumcised & $50(25)$ \\
Planning to be circumcised & $1(1)$ \\
Not circumcised or planning to be circumcised &
\end{tabular}

reported having had no foreskin removed at circumcision, and the genital examination confirmed this; therefore, his data were excluded from the analysis of those who had been circumcised $(n=147)$ (Fig. 1).

Among those who self-reported having been circumcised, the median age at circumcision was 21 years (IQR $19-22$ years). Self-reported circumcision status was positively associated with increasing age (odds ratio (OR) 1.41, 95\% CI 1.27 - 1.58, $p<0.001$ ). There was, however, no association between self-reported circumcision status and religion ( $p=0.31)$ or place of birth $(p=0.60)$. The procedure was performed by 'an old village man' in 135 participants (92\%), by a traditional healer in 9 (6\%), and by a doctor or nurse in $1(0.5 \%)$. (Two people did not answer this question.)

Based on visual genital examination, 107 (75\%) had had a complete circumcision, $24(17 \%)$ had had $40 \%$ or more of the foreskin removed, 10 (7\%) had had less than $40 \%$ removed and 1 participant appeared uncircumcised. Using a kappa analysis, there was only $41 \%$ agreement between the self-reported 'degree of circumcision' and the medical assessment $(p<0.001)$, although the percentage agreement not due to chance was low (14\%).

The median age at which circumcision was planned was 20 years (IQR 19 - 22 years). The procedure was to be performed by 'an old village man' in 47 participants $(94 \%)$ and by a traditional healer in $2(4 \%)$. No participants planned to have a MC and 1 was unsure who would perform the procedure.

Twenty-seven participants (14\%) were HIV-infected, of whom 21 reported having been circumcised. There was no association between HIV status and self-reported circumcision status $(p=0.41)$. Similarly, there was no association between HIV status and medically assessed circumcision status $(p=0.98)$, regardless of whether foreskin removal was deemed to be complete or partial $(p=0.91)$.

One hundred and twenty-eight participants (66\%) were aware that circumcision offered a degree of protection against STIs and HIV: 93 (63\%) of those who self-reported having been circumcised and 35 (70\%) who reported planning circumcision.

The most frequently reported reasons for circumcision were religion ( $86 \%$ of those circumcised and $82 \%$ of those planning circumcision), pleasing parents or family ( $74 \%$ and $20 \%)$, and becoming a man $(33 \%$ and $92 \%)$. Only $3 \%$ of those who self-reported having been circumcised, and $2 \%$ of those who self-reported planning circumcision, endorsed protection against STIs and HIV as a motivator. When asked how they would feel if told by a medical practitioner that they were only partially circumcised, most said they would not care $(n=97,66 \%)$, with some saying they would feel ashamed, depressed or angry $(n=51,34 \%)$.

Among those who self-reported having been circumcised, 127 (86\%) were unwilling to undergo $\mathrm{MC}$ if they learnt that their circumcision had been incomplete; $15(10 \%)$ reported willingness, and $5(4 \%)$ were unsure. Among those who self-reported planning circumcision, 40 (80\%) were unwilling to undergo MC in addition to TC, should the latter be incomplete, while $5(10 \%)$ were willing, and $5(10 \%)$ were unsure. There were no significant differences in willingness to undergo MC between those who self-reported having been circumcised and those who self-reported planning circumcision, although the proportion unsure whether to undergo MC was slightly higher in those who were planning circumcision ( $10 \%$ v. $4 \%, p=0.07$ ).

The most frequently reported reasons for willingness to undergo MC among those self-reporting having been circumcised and those self-reporting planning circumcision were becoming a man $(33 \%$ of those circumcised and $16 \%$ of those planning circumcision) and to be clean/hygienic (30\% and 21\%). The most frequently reported reasons for unwillingness to undergo MC among those who self-reported having been circumcised and those planning circumcision were that it was against their religion or culture $(76 \%$ of those circumcised and $75 \%$ of 


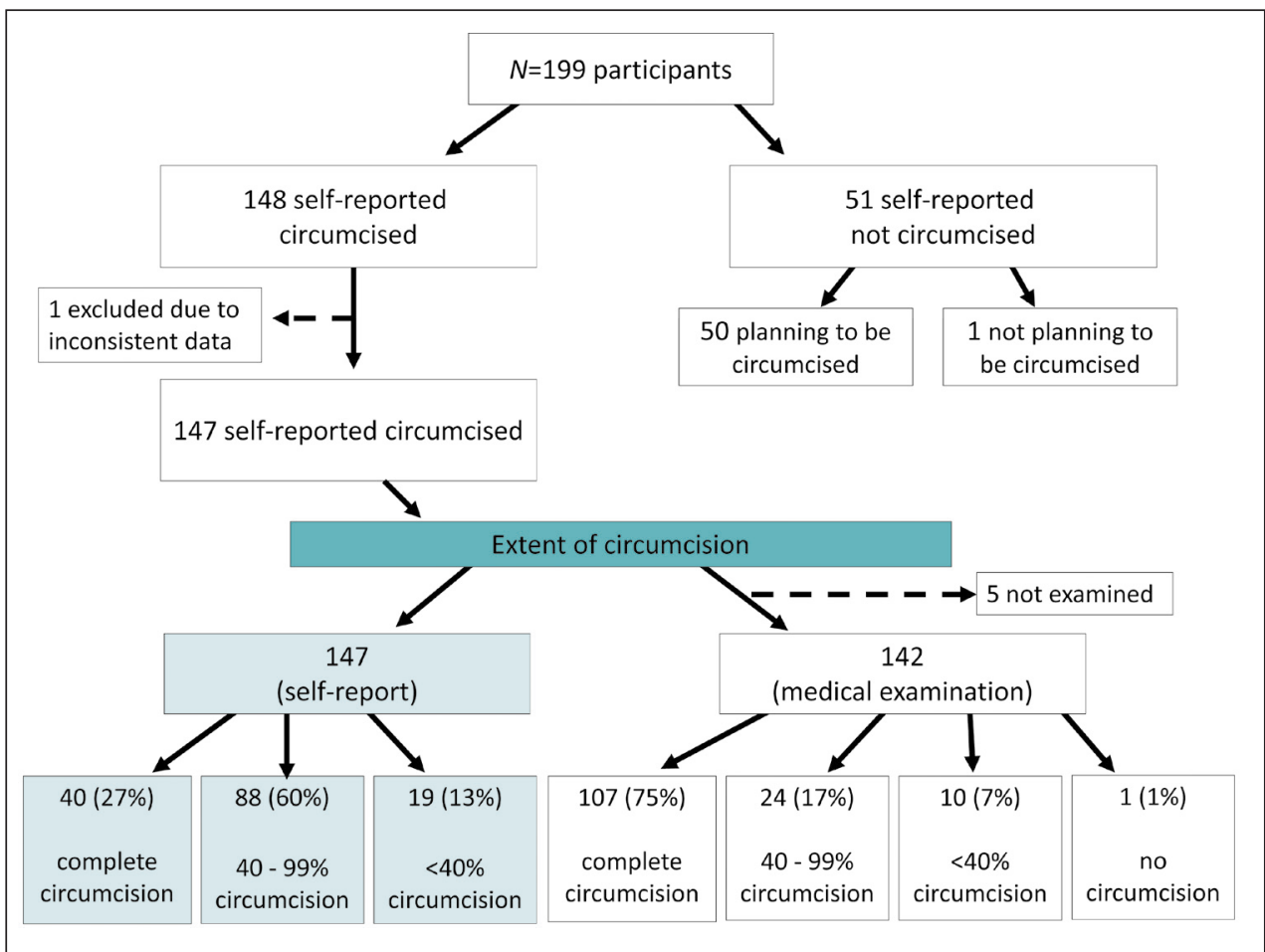

Fig. 1. Cohort diagram.

those planning circumcision), it would make one less of a man ( $42 \%$ and $58 \%$ respectively), and parents, family or community would not approve ( $25 \%$ and $45 \%$ respectively).

A subset of participants $(n=169)$, who self-reported having a son under the age of 15 years $(n=67,40 \%)$ and/or reported planning to have children ( $n=102,60 \%)$, were asked about willingness to allow their son(s) to undergo MC instead of TC. Twenty-seven were willing (16\%), 138 unwilling (82\%) and 4 unsure (2\%). When asked about their willingness to allow their son(s) to undergo $\mathrm{MC}$ in addition to TC, 31 were willing (18\%), 123 unwilling (73\%) and 14 unsure (8\%) (no data for 1 individual). Most felt the decision was theirs to make ( $83 \%$ for the decision to replace TC with MC and $84 \%$ for the decision to have both); some felt it would be a collaborative decision with a partner $(10 \%$ and $8 \%)$, and others said it would be their son's decision (3\%). Among those who reported willingness to allow MC in addition to TC, 23 (75\%) would only allow it in early adulthood (age 18 - 21 years) and $8(25 \%)$ in the early 20 s. None of the participants reported willingness to allow MC in infancy or childhood.

\section{Discussion}

Information around acceptability of $\mathrm{MC}$ in populations that traditionally practise circumcision is limited. In a 2007 review, consistently high levels of uncircumcised men (median 65\%) were reported to be willing to undergo the procedure across sub-Saharan Africa. ${ }^{13}$ Within this study, high rates of realised or planned TC were found. Despite more than two-thirds of the sample demonstrating knowledge regarding the HIV prevention efficacy of circumcision, $\mathrm{MC}$ - and in particular a repeat procedure - was not acceptable.

The discrepancy between sexual debut and time of procedure may jeopardise the potential effectiveness of TC as an HIV prevention strategy. The median age at circumcision was 21 years for this cohort, yet the mean age of sexual debut has been reported to be 14.6 in a similar Cape Town community ${ }^{14}$ and HIV prevalence in those under 20 years was reported to be $14 \%$ in a 2008 national report. ${ }^{2}$ To realise the full benefit of circumcision in reducing HIV acquisition, it would be optimal to ensure that the procedure be carried out before any sexual risk exposure.

In this study more than a quarter of those having undergone TC were left with some foreskin in place. The importance of complete foreskin removal in terms of HIV transmission efficacy is not known. Possible biological explanations for the efficacy of circumcision, including the higher density of HIV target cells on the inner mucosal foreskin surface, susceptibility to abrasions and incidence of ulcerative STIs, would all remain as potential HIV transmission routes with partial foreskin in situ?

Study limitations include the modest cohort size. In addition, the men were recruited from a group volunteering at a prevention research centre and VCT site; therefore, results may not be generalisable. A strength of the study is that the circumcision data were not dependent on selfreport but were confirmed by clinical examination.

Combinations of MC with the other components of the cultural ritual were not extensively dealt with in this study and warrant further research. Perceptions and attitudes on the part of the community that $\mathrm{MC}$ is against tradition and cultural notions of manhood, may need to be targeted in order to facilitate acceptability of MC.

Acknowledgements. We thank the participants and staff at the Emavundleni Prevention Centre. The study was in part funded by the International Aids Vaccine Initiative and the South African Aids Vaccine Initiative.

\section{References}

1. UNAIDS. UNAIDS report on the global AIDS epidemic 2010. Geneva. http://www.unaids.org/ globalreport/global_report.htm (accessed 19 June 2011).

2. Shisana O, Rehle T, Simbayi LC, et al. South African National HIV Prevalence, Incidence, Behaviour and Communication Survey, 2008:A Turning Tide Among Teenagers. Cape Town: HSRC Press, 2009.

3. Auvert B, Taljaard D, Lagarde E, Sobngwi-Tambekou J, Sitta R, Puren A. In: Deeks S, ed. Randomized, controlled intervention trial of male circumcision for reduction of HIV infection risk: the ANRS 1265 trial. PLoS Med 2005;2(11):e298.

Bailey RC, Moses S, Parker CB, et al. Male circumcision for HIV prevention in young men in Kisumu, Kenya: a randomised controlled trial. Lancet 2007;369(9562):643-656.

5. Gray RH, Kigozi G, Serwadda D, et al. Male circumcision for HIV prevention in men in Rakai, Uganda: a randomised trial. Lancet 2007;369(9562):657-666.

Williams BG, Lloyd-Smith JO, Gouws E, et al. The potential impact of male circumcision on HIV in sub-Saharan Africa. PLoS Med 2006;3(7):e262.

Weiss HA, Thomas SL, Munabi SK, Hayes RJ. Male circumcision and risk of syphilis, chancroid, and Weiss HA, Thomas SL, Munabi SK, Hayes RJ. Male circumcision and risk of syphilis, chancroid,
genital herpes: a systematic review and meta-analysis. Sex Transm Infect 2006;82(2):101-110.

8. Connolly C, Simbayi LC, Shanmugam R, Nqeketo A. Male circumcision and its relationship to HIV infection in South Africa: results of a national survey in 2002. S Afr Med J 2008;98(10):789-794.

9. Statistics South Africa. Census 2001: Census in Brief. Pretoria: Statistics South Africa, 2003.

10. Mavundla TR, Netswera FG, Bottoman B, Toth F. Rationalization of indigenous male circumcision as a sacred religious custom: health beliefs of Xhosa men in South Africa. J Transcult Nurs 2009;20(4):395-404.

City of Cape Town. Nyanga district population, 2003. http://www.capetown.gov.za (accessed 27 July 2009).

12. Western Cape Provincial Department of Health. HIV and Syphilis in the Western Cape: Results of the 2008 HIV and Syphilis Antenatal Provincial and Sub-District Surveys. Cape Town: WC Prov Dept of Health, 2008.

3. Westercamp N, Bailey RC. Acceptability of male circumcision for prevention of HIV/AIDS in subSaharan Africa: a review. AIDS Behav 2006;11(3):341-355.

14. Jaspan HB, Berwick JR, Myer L, et al. Adolescent HIV prevalence, sexual risk, and willingness to participate in HIV vaccine trials. J Adolesc Health 2006;39(5),642-648. 